\title{
COMPLICATIONS OF ACUTE MYOCARDIAL INFARCTION RESULTING IN LATE INHOSPITAL MORTALITY
}

\author{
By Khalid H. Sheikh, M.D.*
}

The greatest danger period of an acute myocardial infarction is the time spent after onset of infarction until hospitalization and the first twenty-four hours of hospitalization. Eighty-five percent of all deaths from acute infarction will occur during this period. The overwhelming majority of deaths occur due to lethal arhythmias or cardiogenic shock. For the survivors of the first 24 hours period, the mortality pattern during the remainder of the hospitalization is very different. In a sense, mortality during this time period may be even more tragic, as frequently the patient and family have been reassured that the great danger period has passed and then suddenly the patient is found deteriorating or dead.

Statistically speaking, the assurance that after the first 24 hours of hospitalization, all is likely to be well is unfounded. The mortality from an acute myocardial infarction after hospitalization is generally placed at $10 \%$ to $15 \%$. However, only $35 \%$ of these deaths occur within the first 24 hours, and $46 \%$ occur after the third hospital day. ${ }^{1}$ What are the characteristics of a completed infarction that lead to late hospital mortality? Clinical experience $^{2}$ as well as pathologic studies ${ }^{3}$ show clearly that the likelihood of late complications and death in hospital is related closely and directly to the size of the completed infarct. This is manifested acutely by left ventricular failure. The presence of failure and age of the patient are the most sensitive indicators of prognosis. Although the single most important cause of mortality is massive damage to the left ventricle, the mechanisms of death are diverse. The mechanism can broadly be grouped into electrical complications (ventricular fibrillation and heart block) and mechanical complications (shock and cardiac rupture) as well as a few miscellaneous causes such as pulmonary embolus.

\section{Arhythmias}

Ventricular fibrillation occurs in $5 \%$ to $10 \%$ of uncomplicated infarctions and a significantly higher percentage of complicated infarcts. Warning

\footnotetext{
*Resident in Medicine University of Colorado, Denver, Co.
}

arhythmias occur in only $50 \%$ of patients with ventricular fibrillation in time to initiate therapy. Prophylactic lidocaine during the first 24 to 48 hour period is standard therapy at many institutions in all patients with a high index of suspicion for acute infarction and without a contraindication. Identifying patients at risk for late arhythmias is more difficult.

Ventricular fibrillation occurring after the initial 24 hour period carries a poor prognosis. It most frequently presents as sudden death and has been shown to be responsible for $7 \%$ of all late inhospital deaths from an acute infarction. ${ }^{4}$ Late fibrillation occurs more frequently in anterior infarctions, large infarctions, in the presence of heart failure and in patients with a history of major arhythmias. In a series of consecutive patients admitted with acute infarction, $3 \%$ will have late inhospital ventricular fibrillation. ${ }^{5}$ This group had a mortality rate of $60 \%$ and almost half had suffered an anteroseptal infarct, complicated by right or left bundle branch block. When this group with anteroseptal infarction and right or left bundle branch block were prospectively studied, with continuous electrical monitoring for six weeks after infarction, $36 \%$ sustained late in-hospital ventricular fibrillation and had a $35 \%$ mortality rate. This may then appear to be a potentially high risk group which would benefit from more prolonged monitoring and pre-discharge holter monitoring with aim toward institution of antiarhythmic therapy.

\section{Ventricular conduction defects complicate as} many as $20 \%$ of all cases of acute myocardial infarction. ${ }^{6}$ Patients developing conduction defects have a mortality rate twice as high as those without conduction defects. A variety of bundle branch blocks have been recognized as precursors to complete heart block. Whereas conduction defects in inferior infarctions are usually hemodynamically not significant, transient and carry little morbidity, those occuring in anterior infarctions identify a group of patients at high risk for hemodynamic instability, complications and death.

Particularly ominous is development of right bundle branch block, which signifies severe 
myocardial damage and carries a rate of $30 \%$ for late in-hospital death. When associated with left fasicular blocks or alternating bundle branch block, there is a high likelihood that complete heart block will develop and has been cited as indications for temporary pacemaker insertion. It is of interest however that the mechanism of death in these patients is nearly always a result of cardiogenic shock secondary to massive damage of the anterior ventricular wall or ventricular fibrillation causing sudden death. Death is only rarely due to asystole associated with complete heart block. Thus, prophylactic pacing has not been shown to improve survival in patients with ventricular conduction defects. The significance of recognizing high risk conduction defects may then be to identify a group of patients at high risk for complications which may be averted by prolonged electrical monitoring or earlier use of hemodynamic monitoring.

\section{Cardiogenic Shock}

Pump failure accounts for greater than $60 \%$ of all deaths from acute infarction. Aside from massive ventricular damage, a number of clinical syndromes can manifest themselves as cardiogenic shock. These include pulmonary embolus, dissection, mitral regurgitation, ventricular septal rupture and right ventricular infarction. These etiologies should be identified to target specific therapy. When none of the above syndromes is evident, one is left with a diagnosis of cardiogenic shock secondary to massive infarction. In this situation it is essential to institute hemodynamic monitoring to guide therapy. Use of the swan-ganz catheter can help identify hemodynamic subsets, as outlined by Swan and Forrester ${ }^{7}$ to guide therapy. Based on the pulmonary capillary wedge pressure and cardiac index (which reflect the degree of pulmonary congestion and peripheral perfusion, respectively) therapy can be initiated and followed and prognosis can be estimated.

Class I represents normal wedge pressure and normal cardiac index. No specific therapy is required and the overall mortality is $1 \%$.

Class II represents pulmonary congestion without peripheral hypoperfusion (pulmonary edema). The wedge pressure is elevated but cardiac index is normal. This usually responds to therapy with oxygen, diuretics, morphine, tourniquets and phlebotomy. Digoxin is not indicated in the acute management. Refractory pulmonary congestion may be treated with nitroglycerin or nitroprusside. Mortality in this group is $11 \%$.

Class III represents peripheral hypoperfusion. The cardiac index is low but wedge pressure is not elevated. Management consists of optimizing filling
FORRESTER HEMODYNAMIC CLASSIFICATION

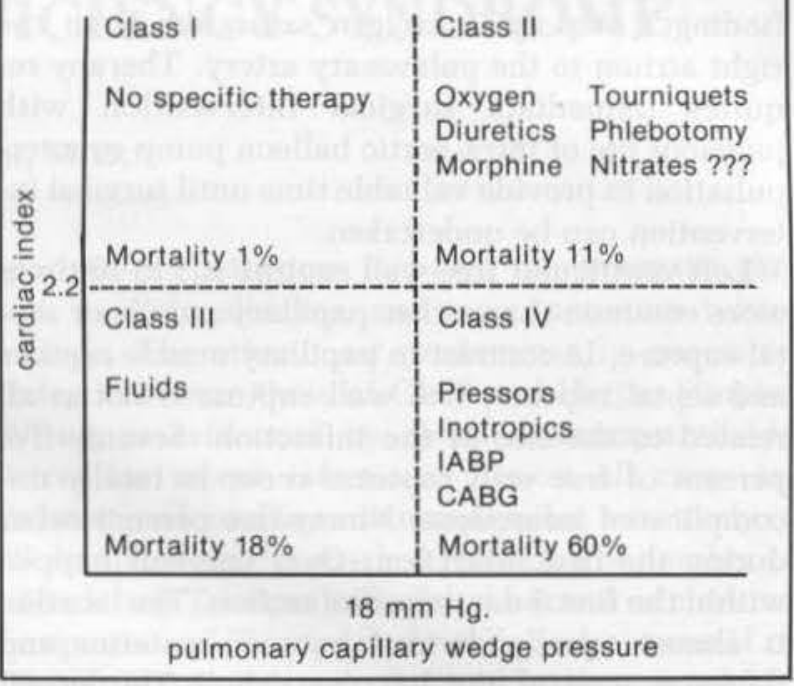

pressure to $15 \mathrm{~mm} \mathrm{Hg}$. to $18 \mathrm{~mm} \mathrm{Hg}$. by the judicious use of fluids. If the wedge pressure cannot be increased, the patient may have a right ventricular infarction. Mortality in this class is $18 \%$.

Class IV occurs if the wedge pressure is increased to $18 \mathrm{~mm} \mathrm{Hg}$. or greater with persistent hypoperfusion, and signifies cardiogenic shock. Management consists of correcting other possible causes of hypotension (anemia, sepsis, pulmonary embolus, hypoxemia, drugs, electrolyte and acid-base abnormalities, arhythmias) and then institution of therapy with pressors and inotropic agents. Most cardiologists advocate early use of the intra-aortic balloon pump (IABP) if the patient is considered a surgical candidate, with aim towards cardiac catheterization as a prelude towards coronary artery bypass grafting (CABG). Mortality in this group is over $60 \%$.

\section{Cardiac Rupture}

Rupture of the myocardium accounts for $5 \%$ to $24 \%$ of late in-hospital mortality from acute myocardial infarction. ${ }^{8}$ Rupture occurs in $1 \%$ to $3 \%$ of patients with acute infarction. ${ }^{2}$ Rupture typically occurs in one of three locations. Rupture of the papillary muscle and interventricular septum is relatively rare and occurs in $1 \%$ of autopsy studies. Both types of rupture are associated with massive infarctions and are manifested by sudden pump failure, usually occurring within the first 3 to 7 days of infarction. Both must be considered in the differential diagnosis of a new harsh systolic murmur in the setting of an acute infarction. The prognosis is poor in either case. The diagnosis is rarely made antemortem. The presence of a swan-ganz catheter may aid in diagnosis. Papillary muscle rupture, resulting in acute mitral regurgitation may be 
heralded by the presence of dominant $\mathrm{V}$ waves. Ventricular septal rupture may be diagnosed by finding a step-up in oxygen saturation from the right atrium to the pulmonary artery. Therapy requires immediate surgical intervention with judicious use of intra-aortic balloon pump counterpulsation to provide valuable time until surgical intervention can be undertaken.

Left ventricular free wall rupture is 8 to 10 times more common than either papillary muscle or septal rupture. In contrast to papillary muscle rupture and septal rupture, free wall rupture is not at all related to the size of the infarction. Seventy-five percent of free wall ruptures occur in totally uncomplicated infarctions. Ninety-five percent occur during the first infarction. Over one-half happen within the first 5 days after infarction. The location is almost equally divided between anterior and posterior wall of the left ventricle. ${ }^{10}$ The lateral wall and apex are uncommonly involved. Free wall rupture occurs more frequently in older patients and relatively more frequently in women. The lone clinical factor to be identified with an increased likelihood of rupture is the presence of postinfarction hypertension. Presentation is typically with sudden dyspnea, pain, hypotension, bradycardia and electromechanical dissociation.

Contrary to what is commonly thought, rupture does not appear to be a "blow-out" phenomenon. Two facts support this. First, there is no relation between infarct size and free wall rupture. Secondly, rupture is quite common within the first 24 hours of infarction, by which time there presumable is not enough myocardial necrosis to result in weakening of the ventricular wall. Rupture may in fact be an insidious event, characterized by successive leaks of small amounts of blood into the pericardial cavity. When enough blood has accumulated to cause hemodynamic compromise, the patient begins to have symptoms which progress rapidly unless pericardial fluid is withdrawn. Indeed, therapy consists of immediate pericardiocentesis. One need not have to remove more than $30 \mathrm{cc}$. to $50 \mathrm{cc}$. of fluid to relieve the acute problem. The patient should be supported with aggressively administered fluids until emergent surgery can be performed. The mortality is expectedly high.

\section{Pulmonary Embolus}

This is responsible for $1 \%$ of deaths during an acute infarction in the late hospitalization phase. Since most emboli arise from thromboses in the lower extremities, it is didactic to look at the risk of deep venous thromboses in the setting of an acute infarction. Up to $40 \%$ of patients may develop calf vein thrombosis in the setting of an acute infarc- tion." Spread to thigh may occur in up to $15 \%$ to $20 \%$ of these patients. Thus, a risk for pulmonary embolization would appear to exist for approximately $8 \%$ to $10 \%$ of all patients with acute infarction. Patients said to be at higher risk are those with a previous history of thrombophlebitis or embolism, those at prolonged bedrest and those with clinically evident congestive heart failure. Overall risk may be lowered by early ambulation and the use of minidose subcutaneous heparin therapy for patients at bedrest. High risk groups, as identified above, should be considered for prophylactic full dose intravenous heparinization during the high risk period.

\section{Conclusion}

Although death from ischemic heart disease occurs in the majority of cases within 24 hours of a new clinical event, up to $50 \%$ of deaths can occur after the first 48 hours of hospitalization. Frequently, these deaths may occur unexpectedly and during the supposed "rehabilitation" period. It is useful to be aware of the syndromes which can result in late in-hospital mortality and be able to identify those patients at high risk. It is only then that appropriate measures can be taken to more closely monitor these patients and intervene with therapy earlier to improve survival.

\section{References}

1. Norris R, et al; Predictors of Late Hospital Death in Acute Myocardial Infarction. Progress in Cardiovascular Diseases, Vol. XXIII, No. 2:129, 1980.

2. Sobel B, et al; Estimation of Infaret Size in Man and Its Relation to Prognosis. Circulation 46:640, 1972.

3. Alonso, D, et al; Pathophysiology of Cardiogenis Shock. Quantification of Myocardial Necrosis, Clinical, Pathological and Electrocardiographic Correlation. Circulation 48:588, 1973.

4. Norris R, et al; Prognostic Factors in Myocardial Infarction. British Medical Journal 3:143, 1968.

5. Lie K, et al; Early Identification of Patients Developing Late In Hospital Ventricular Fibrillation after Discharge from the CCU. American Journal of Cardiology 41:674, April 1978.

6. Atkins J, et al; Ventricular Conduction Blocks and Sudden Death in Acute Myocardial Infarction. NEJM 228 (6):281, February 1973.

7. Forrester J, et al; Medical Therapy of Acute Myocardial Infarction by Application of Hemodynamic Subsets. NEJM 295 (24 \& 25): 1356 \& 1404, December 1976.

8. Nicod P, et al; Myocardial Rupture after Myocardial Infarction. American Journal of Medicine 73:765, November 1982.

9. Naeim F; et al; Cardiac Rupture During Myocardial Infarction. Circulation. 45:1231, June 1972.

10. Baker R, et al; Cardiac Rupture-Challenge in Diagnosis and Management. American Journal of Cardiology 40:429, September 1977.

11. Maurer B, et al; Frequency of Venous Thrombosis after Myocardial Infarction. Lancet. 2:1385, 1971. 